\title{
AN ASSORTMENT OF NEGATIVELY CURVED ENDS
}

\author{
IGOR BELEGRADEK
}

\begin{abstract}
Motivated by recent groundbreaking work of Ontaneda, we describe a sizable class of closed manifolds such that the product of each manifold in the class with $\mathbb{R}$ admits a complete metric of bounded negative sectional curvature which is an exponentially warped near one end and has finite volume near the other end.
\end{abstract}

\section{INTRODUCTION}

Let $V$ be a finite volume, complete, open Riemannian manifold of sectional curvature $K$ within $[-1,0)$. Little is known about the topology of $V$. An argument of Gromov [Gro78] extended by Schroeder [BGS85, Appendix 2] implies that $V$ is diffeomorphic to the interior of a compact manifold $\bar{V}$ with boundary; thus any manifold compactification of $V$ is obtained by attaching an h-cobordism to $\bar{V}$ along $\partial \bar{V}$.

A well-known problem is to determine which manifolds occur as the boundary of $\bar{V}$. Gromov showed that $\partial \bar{V}$ must have zero simplicial volume Gro82, p.37], and this seems to be the only known obstruction in the case when each component of $\partial \bar{V}$ is aspherical. Other obstructions were found in $\mathrm{BP}$ when $\partial \bar{V}$ has a non-aspherical component, denoted $C$; e.g. $\pi_{1}(C)$ cannot be an irreducible, higher rank lattice, or a virtually nilpotent group.

Earlier examples of manifolds appearing as components of $\partial \bar{V}$ include generalized graph manifolds [AS92, Buy93, and some circle bundle over real and complex hyperbolic manifolds [Fuj88, Bel12b, Bel12a]. A recent breakthrough of Ontaneda allows to dramatically expand the list.

If a (not necessarily connected) manifold $B$ is diffeomorphic to the boundary of a connected, smooth, compact manifold $N$, then we say that $B$ bounds $N$, and if $N$ is not specified, we simply say $B$ bounds.

Ontaneda's proof starts with a closed manifold $B$ that bounds, and applies relative strict hyperbolization of Charney-Davis CD95 to realize $B$ as a boundary of a compact manifold whose interior admits a piecewise hyperbolic metric.

2000 Mathematics Subject classification. Primary 53C20. Keywords: negative curvature, end, warped product, finite volume. 
Then under the assumption that the building block in the hyperbolization is "large enough" Ontaneda is able to smooth the metric away from the boundary to a Riemannian metric with $K$ near -1 ; this smoothing argument is technological tour de force. Near the boundary the metric has to be constructed by an ad hoc method depending on $B$. The following result is implicit in Ont.

Theorem 1.1. (Ontaneda) Let $B$ be a closed $n$-manifold that bounds and satisfies the following:

(i) If $n \geq 5$, then any $h$-cobordism from $B$ to another manifold is a product.

(ii) $\mathbb{R} \times B$ admits a complete metric $g$ of sectional curvature within $[-1,0)$ such that $(-\infty, 0] \times B$ has finite $g$-volume, and $g=d r^{2}+e^{2 r} g_{B}$ on $[c, \infty) \times B$ for some $c>0$ and a metric $g_{B}$ on $B$.

Then $B$ bounds a manifold whose interior admits a complete metric of finite volume and sectional curvature in $[-1,0)$.

Condition (ii) implies that each component of $B$ is aspherical, and hence has torsion-free fundamental group. The Whitehead Torsion Conjecture, which is true for many groups of geometric origin, predicts that all torsion-free groups have zero Whitehead torsion. If the conjecture is true for the fundamental group of each component of $B$, then (i) holds.

For example (ii) is true if $B$ is any infranilmanifold [BK05, or any 3 -dimensional Sol manifolds [Pha]. We add to the list as follows.

Theorem 1.2. Condition (ii) holds for every manifold in the class $\mathcal{B}$ that is defined as the smallest class of closed manifolds of positive dimension such that

- $\mathcal{B}$ contains each infranilmanifold, every circle bundle of type $(\mathrm{K})$, and each closed manifold of $K \leq 0$ with a local Euclidean de Rham factor;

- $\mathcal{B}$ is closed under products, disjoint unions, and products with any compact manifold of $K \leq 0$.

An orientable circle bundle has type $(\mathrm{K})$ if its base is a closed complex hyperbolic $n$-manifold $M$ whose holonomy representation $\pi_{1}(M) \rightarrow P U(n, 1)$ lifts to $U(n, 1)$, and if the Euler class of the bundle equals $-m \frac{\omega}{4 \pi}$ for some nonzero integer $m$, where $\omega$ is the Kähler form of $M$. For example, each nontrivial orientable circle bundle over a genus two orientable closed surface has type $(\mathrm{K})$. It is immediate from $[\mathrm{BL}, \mathrm{BFL}$ that (i) holds for every manifold in $\mathcal{B}$, so Theorems 1.11 .2 imply

Corollary 1.3. If a manifold $B$ in $\mathcal{B}$ bounds, then $B$ bounds a compact connected manifold whose interior $V$ admits a complete Riemannian metric of finite volume and sectional curvature in $[-1,0)$.

Theorem 1.2 is proved by showing that each manifold in $\mathcal{B}$ carries what we call a simultaneously diagonalizable family of metrics $g_{r}$ such that $g=d r^{2}+g_{r}$ 
is as in (ii), and the main observation is that simultaneous diagonalizability behaves well under products, and facilitates curvature computations.

Results of this paper can be modified to hold for other curvature conditions such as $K \leq-1$, or $-1 \leq K \leq 0$, and other growth assumptions on the ends, such as infinite volume or Rad Inj $\rightarrow 0$, which is all left to an interested reader to explore.

Acknowledgments The author is grateful for NSF support (DMS-1105045).

\section{Curvature formulas}

Let us review the curvature formulas for the metric $g=d r^{2}+g_{r}$ on $I \times B$ that were derived in [BW04, Section 6] and corrected in [Bel12b, Appendix C], where $I$ is an open interval, and $B$ is a manifold. The family of metrics $\left(B, g_{r}\right), r \in I$ is called simultaneously diagonalizable if near each point of $B$ there is a basis of vector fields $\left\{X_{i}\right\}$ that is $g_{r}$-orthogonal for each $r$. Set $h_{i}(r)=\sqrt{g_{r}\left(X_{i}, X_{i}\right)}$ and note that $Y_{i}=X_{i} / h_{i}$ form a $g_{r}$-orthonormal basis. Denote $g(X, Y)$, $\frac{\partial}{\partial r}$ by $\langle X, Y\rangle, \partial_{r}$, respectively. If $g_{r}$ are simultaneously diagonalizable, the components of the curvature tensor of $g=d r^{2}+g_{r}$ are

$$
\begin{gathered}
(2.1) \quad\left\langle R_{g}\left(Y_{i}, Y_{j}\right) Y_{j}, Y_{i}\right\rangle=\left\langle R_{g_{r}}\left(Y_{i}, Y_{j}\right) Y_{j}, Y_{i}\right\rangle-\frac{h_{i}^{\prime} h_{j}^{\prime}}{h_{i} h_{j}}, \\
(2.2) \quad\left\langle R_{g}\left(Y_{i}, Y_{j}\right) Y_{l}, Y_{m}\right\rangle=\left\langle R_{g_{r}}\left(Y_{i}, Y_{j}\right) Y_{l}, Y_{m}\right\rangle \quad \text { if }\{i, j\} \neq\{l, m\}, \\
(2.3) \quad\left\langle R_{g}\left(Y_{i}, \partial_{r}\right) \partial_{r}, Y_{i}\right\rangle=-\frac{h_{i}^{\prime \prime}}{h_{i}}, \quad\left\langle R_{g}\left(Y_{i}, \partial_{r}\right) \partial_{r}, Y_{j}\right\rangle=0 \quad \text { if } i \neq j \\
2\left\langle R_{g}\left(\partial_{r}, Y_{i}\right) Y_{j}, Y_{k}\right\rangle= \\
\left.(2.4) \quad\left\langle Y_{i}, Y_{j}\right], Y_{k}\right\rangle\left(\ln \frac{h_{k}}{h_{j}}\right)^{\prime}+\left\langle\left[Y_{k}, Y_{i}\right], Y_{j}\right\rangle\left(\ln \frac{h_{j}}{h_{k}}\right)^{\prime}+\left\langle\left[Y_{k}, Y_{j}\right], Y_{i}\right\rangle\left(\ln \frac{h_{i}^{2}}{h_{j} h_{k}}\right)^{\prime}
\end{gathered}
$$

The second fundamental form $\mathbb{I}_{g_{r}}$ of $g_{r}$ is given by $\mathbb{I}_{g_{r}}\left(Y_{i}, Y_{j}\right)=0$ if $i \neq j$, and $\mathbb{I}_{g_{r}}\left(Y_{i}, Y_{i}\right)=-\frac{h_{i}^{\prime}}{h_{i}} \partial_{r}$ [BW04].

\section{Products}

Given manifolds $B_{1}, B_{2}$ consider the metrics $d r^{2}+g_{r, k}$ on $\mathbb{R} \times B_{k}$ with $k=1,2$, and $g=d r^{2}+g_{r}$ on $\mathbb{R} \times B_{1} \times B_{2}$ where $g_{r}=g_{r, 1}+g_{r, 2}$. Denote the negative reals by $\mathbb{R}_{-}$.

Theorem 3.1. For $k=1,2$ suppose that $g_{r, k}$ is simultaneously diagonalizable, and $\mathbb{I}_{g_{r}}$ is negative definite for all $r$. Then

(1) $K_{g}<0$ if $K_{d r^{2}+g_{r, 1}}, K_{d r^{2}+g_{r, 2}}$ are negative.

(2) $K_{g}$ is bounded if $K_{d r^{2}+g_{r, 1}}, K_{d r^{2}+g_{r, 2}}, \mathbb{I}_{g_{r}}$ are bounded.

(3) $\operatorname{Vol}\left(\mathbb{R}_{-} \times B_{1} \times B_{2}, g\right)$ is finite if $\operatorname{Vol}\left(\mathbb{R}_{-} \times B_{1}, d r^{2}+g_{r, k}\right)$ and $\operatorname{Vol}\left(B_{2}, g_{0,3-k}\right)$ are finite for some $k$. 
Remark 3.2. If $\mathbb{I}_{g_{r}}$ is positive definite, the same result holds with $\mathbb{R}_{\text {- replaced }}$ by the positive reals.

Proof. The product $g_{r}=g_{r, 1}+g_{r, 2}$ is simultaneously diagonalizable, so let $Y_{i}$ be the corresponding basis vectors tangent to one of the factors. For brevity denote $g\left\langle R_{g}(A, B) C, D\right\rangle$ by $(A, B, C, D)_{g}$. Fix vectors $C, D$, and write $C=C_{1}+C_{2}$, $D=D_{1}+D_{2}$ where $C_{k}, D_{k}$ are tangent to $B_{k}$. Fix reals $a, b$ such that $a \partial_{r}+C, b \partial_{r}+D$ are orthonormal.

Think of $g$ is the Riemannian submersion metric with base $d r^{2}+g_{r, k}$ and fiber $g_{r, 3-k}$. Since the submersion metric $g$ is a warped product, its $A$-tensor vanishes [Bes08, 9.26], so by O'Neill's formula [Bes08, 9.28f] $R_{g}$ restricted to the horizontal space equals $R_{d r^{2}+g_{r, k}}$, and hence the $g$-sectional curvature of any horizontal plane is negative under the assumptions of (1) and bounded under the assumptions of (2). Thus if we show that

$$
\left(a \partial_{r}+C, b \partial_{r}+D, b \partial_{r}+D, a \partial_{r}+C\right)_{g} \leq \sum_{k=1}^{2}\left(a \partial_{r}+C_{k}, b \partial_{r}+D_{k}, b \partial_{r}+D_{k}, a \partial_{r}+C_{k}\right)_{g}
$$

then (1) would follow. Now $\left(a \partial_{r}+C, b \partial_{r}+D, b \partial_{r}+D, a \partial_{r}+C\right)_{g}$ equals

$$
\begin{gathered}
2 a b\left(\partial_{r}, D, \partial_{r}, C\right)_{g}+a^{2}\left(\partial_{r}, D, D, \partial_{r}\right)_{g}+2 a\left(\partial_{r}, D, D, C\right)_{g}+ \\
b^{2}\left(C, \partial_{r}, \partial_{r}, C\right)_{g}+2 b\left(C, \partial_{r}, D, C\right)_{g}+(C, D, D, C)_{g}
\end{gathered}
$$

and each $\left(a \partial_{r}+C_{k}, b \partial_{r}+D_{k}, b \partial_{r}+D_{k}, a \partial_{r}+C_{k}\right)_{g}$ has a similar decomposition into six summands. The desired inequality is to be established one summand at a time, and in fact, it is an equality except for the last summand.

As $\left(\partial_{r}, Y_{i}, \partial_{r}, Y_{j}\right)_{g}=0$ for $i \neq j$ we see that $\left(\partial_{r}, D, \partial_{r}, C\right)_{g}=\left(\partial_{r}, D_{1}, \partial_{r}, C_{1}\right)_{g}+$ $\left(\partial_{r}, D_{2}, \partial_{r}, C_{2}\right)_{g}$, and similar equalities hold for $\left(\partial_{r}, D, D, \partial_{r}\right)_{g},\left(C, \partial_{r}, \partial_{r}, C\right)_{g}$. As $\left(\partial_{r}, Y_{i}, Y_{j}, Y_{k}\right)_{g}=0$ unless $Y_{i}, Y_{j}, Y_{k}$ are tangent to the same factor, we deduce $\left(\partial_{r}, D, D, C\right)_{g}=\left(\partial_{r}, D_{1}, D_{1}, C_{1}\right)_{g}+\left(\partial_{r}, D_{2}, D_{2}, C_{2}\right)_{g}$; a similar formula holds for $\left(C, \partial_{r}, D, C\right)_{g}$.

The last summand is given by the Gauss formula

$$
(C, D, D, C)_{g}=(C, D, D, C)_{g_{r}}+\left\langle\mathbb{I}_{g_{r}}(C, D), \mathbb{I}_{g_{r}}(C, D)\right\rangle_{g}-\left\langle\mathbb{I}_{g_{r}}(C, C), \mathbb{I}_{g_{r}}(D, D)\right\rangle_{g}
$$

and again a similar formula holds for $\left(C_{k}, D_{k}, D_{k}, C_{k}\right)_{g}$.

Since $g_{r}$ is the product metric, $\left(C_{i}, D_{j}, D_{k}, C_{m}\right)_{g_{r}}=0$ unless $i, j, k, l$ are all equal, so $(C, D, D, C)_{g_{r}}=\left(C_{1}, D_{1}, D_{1}, C_{1}\right)_{g_{r}}+\left(C_{2}, D_{2}, D_{2}, C_{2}\right)_{g_{r}}$. Let $T$ be the diagonal matrix with $i i$ entry equal to $\sqrt{\frac{\left|h_{i}^{\prime}\right|}{h_{i}}}$. Since $\mathbb{I}_{g_{r}}$ is nonpositive definite, $\langle T A, T B\rangle_{g} \partial_{r}=-\mathbb{I}_{g_{r}}(A, B)$, so $\left\langle\mathbb{I}_{g_{r}}(C, D), \mathbb{I}_{g_{r}}(C, D)\right\rangle_{g}-\left\langle\mathbb{I}_{g_{r}}(C, C), \mathbb{I}_{g_{r}}(D, D)\right\rangle_{g}=\langle T C, T D\rangle_{g}^{2}-\langle T C, T C\rangle_{g}\langle T D, T D\rangle_{g}$. 
The same formula holds for $C_{k}, D_{k}$ in place of $C, D$. Combining the above with vanishing of $\left\langle T C_{i}, T C_{j}\right\rangle_{g},\left\langle T D_{i}, T D_{j}\right\rangle_{g},\left\langle T D_{i}, T C_{j}\right\rangle_{g}$ for $i \neq j$ gives

$$
\begin{gathered}
(C, D, D, C)_{g}-\left(C_{1}, D_{1}, D_{1}, C_{1}\right)_{g}-\left(C_{2}, D_{2}, D_{2}, C_{2}\right)_{g}= \\
\left(\left\langle T C_{1}, T D_{1}\right\rangle_{g}+\left\langle T C_{2}, T D_{2}\right\rangle_{g}\right)^{2}-\left(\left|T C_{1}\right|_{g}^{2}+\left|T C_{2}\right|_{g}^{2}\right)\left(\left|T D_{1}\right|_{g}^{2}+\left|T D_{2}\right|_{g}^{2}\right) \leq \\
\left(\left|T C_{1}\right|_{g}\left|T D_{1}\right|_{g}+\left|T C_{2}\right|_{g}\left|T D_{2}\right|_{g}\right)^{2}-\left(\left|T C_{1}\right|_{g}^{2}+\left|T C_{2}\right|_{g}^{2}\right)\left(\left|T D_{1}\right|_{g}^{2}+\left|T D_{2}\right|_{g}^{2}\right)= \\
-\left(\left|T C_{1}\right|_{g}\left|T D_{2}\right|_{g}-\left|T C_{2}\right|_{g}\left|T D_{1}\right|_{g}\right)^{2} \leq 0 .
\end{gathered}
$$

which completes the proof of (1).

To prove (2) note that the above inequalities are equalities except in one case where the difference is controlled by $\max _{i, r} \frac{\left|h_{i}^{\prime}\right|}{h_{i}}$, which is bounded. Finally $\left(a \partial_{r}+C_{k}, b \partial_{r}+D_{k}, b \partial_{r}+D_{k}, a \partial_{r}+C_{k}\right)_{g}$ is the product of two bounded quantities, $g$-area of the parallelogram and $d r^{2}+g_{k, r}$-sectional curvature of the plane each spanned by $\left\{a \partial_{r}+C_{k}, b \partial_{r}+D_{k}\right\}$, so (2) follows.

Since $\mathbb{I}_{g_{r}}$ is nonpositive definite, each $h_{i}$ is nondecreasing, so the identity map $\left(B_{k}, g_{0}\right) \rightarrow\left(B_{k}, g_{r, k}\right)$ with $r<0$ is 1 -Lipschitz, and hence volume nonincreasing. Thus if $B_{k}$ has finite $g_{0, k}$-volume, then the $g_{r, k}$-volume of $B_{k}$ is uniformly bounded on $\mathbb{R}_{-}$. Now (3) follows from the Fubini theorem for the Riemannian submersion metric $g$ with base $d r^{2}+g_{r, k}$ and fiber $g_{r, 3-k}$.

\section{Nonpositively CURVEd MANIFOLDS}

Here is a souped up version of the fact that $d r^{2}+e^{2 r} g_{B}$ has $K<0$ whenever $K_{g_{B}} \leq 0$.

Theorem 4.1. If $\left(B, g_{B}\right)$ is a manifold of bounded nonpositive curvature, then there is a convex, increasing, smooth, positive function $h$ that equals $e^{r}$ for large $r$, and such that the sectional curvature $\left(\mathbb{R} \times B, d r^{2}+h^{2} g_{B}\right)$ is negative and bounded below.

Proof. Any 2-plane tangent to $\mathbb{R} \times B$ is of the form $\operatorname{span}\left\{X_{1}, c X_{2}+d \partial_{r}\right\}$ where each $\left\{X_{1}, X_{2}\right\}$ are $g_{B}$-orthonormal vectors tangent to the $B$-factor and $c^{2}+d^{2}=1$. Let $Y_{i}:=X_{i} / h$, so that $Y_{1}, Y_{2}, \partial_{r}$ is $g$-orthonormal, where $g=d r^{2}+h^{2} g_{B}$. Then $\left\langle R_{g}\left(Y_{i}, Y_{j}, \partial_{r}\right) Y_{k}\right\rangle_{g}=0$ and

$$
K_{g}\left(Y_{1}, c Y_{2}+d \partial_{r}\right)=c^{2}\left(\frac{K_{g_{B}}\left(Y_{1}, Y_{2}\right)}{h^{2}}-\left(\frac{h^{\prime}}{h}\right)^{2}\right)-d^{2} \frac{h^{\prime \prime}}{h} .
$$

which is negative if $h^{\prime}, h^{\prime \prime}$ are negative and $K_{g_{B}} \leq 0$. To make $K_{g}$ bounded below fix any $\tau>0$, and let $h$ be an increasing strictly convex function such that $h(r)=e^{r}+\tau$ for $r<0$ and $h(r)=e^{r}$ for $r \geq r_{\tau}$, which exists is $r_{\tau}$ is 
large enough. (e.g. $h$ can be obtained by smoothing the function that equals $e^{r}+\tau$ for $r<0$, equals $r+1+\tau$ until the line intersects the graph of $e^{r}$, and equals $e^{r}$ after that). Then $K_{g}\left(Y_{1}, c Y_{2}+d \partial_{r}\right)$ is negative and bounded.

Corollary 4.2. Let $\left(B, g_{B}\right)$ be a compact manifold of nonpositive curvature whose universal cover has a Euclidean de Rham factor. Then $B$ has a simultaneously diagonalizable family of metrics $g_{r}$ such that $g_{r}=e^{2 r} g_{B}$ for large $r$, $K_{d r^{2}+g_{r}}$ is negative and bounded below, and $\operatorname{Vol}\left(\mathbb{R}_{-} \times B, d r^{2}+g_{r}\right)$ is finite.

Proof. Let $\left(\mathbb{R}^{s}, g_{0}\right) \times\left(X, g_{X}\right)$ be a de Rham splitting of the universal Riemannian cover $\left(\tilde{B}, \tilde{g}_{B}\right)$ of $\left(B, g_{B}\right)$, where $g_{0}$ is the standard Euclidean metric, $s>0$, and $X$ has no Euclidean factors. Consider the metric $\tilde{g}=d r^{2}+e^{2 r} g_{0}+h^{2} g_{X}$ on $\mathbb{R} \times \tilde{B}$ where $h$ is as in Theorem 4.1, and by that theorem $K_{\tilde{g}}$ is negative and bounded below. Since the de Rham decomposition is invariant under isometries, the metric descends to a metric $d r^{2}+g_{r}$ on $\mathbb{R} \times B$ with the same curvature bounds. By compactness $B$ is covered by finitely many "product charts" that lift isometrically to the universal cover to the product of balls in $\mathbb{R}^{s}$ and $X$. The product of any such chart with $\mathbb{R}_{-}$has finite $g$-volume, and hence so does $\mathbb{R}_{-} \times B$.

\section{INFRANILMANIFOLDS}

Let $B$ be an infranilmanifold of nilpotence degree $k$. The metric on $B$ constructed [BK05] can be modified to satisfy Theorem 1.1](ii), while keeping the sectional curvature almost in $\left[-k^{2},-1\right]$. This was used but not explained in Ont, so we supply a proof below. Here "almost" means that for any $\varepsilon>0$ there is a metric as in (ii) with sectional curvature within $\left[-(k+\varepsilon)^{2},-1\right]$.

The metric in BK05] is of the form $g=d r^{2}+g_{r}$, where $g_{r}$ is simultaneously diagonalizable, and the only part of (ii) that does not hold for $g$ is that for all $r \geq c$ we have $g_{r}=e^{2 k r} g_{B}$ where $g_{B}$ is a fixed metric. To satisfy (ii) we need to "replace" $k$ by 1 .

To do so choose a non-increasing function $Q$ of $r$ such that $Q(r)=k$ on $\left[c, T_{1}\right]$ and $Q(r)=1$ for $r \geq T_{2}$. By making $T_{2} \gg T_{1}$ we may assume that $Q^{\prime}$ is uniformly small. Set $q:=k c+\int_{c}^{r} Q(r) d r$ so that $q(r)=k r$ on $\left[c, T_{1}\right]$ and $q(r)=r+(k-1) c$ for $r \geq T_{2}$. Set $h:=e^{q}$ and consider the metric $\bar{g}:=d r^{2}+\bar{g}_{r}$ such that $\bar{g}_{r}=g_{r}$ for $r \leq T_{1}$, and $\bar{g}_{r}=h^{2} g_{B}$ for $r \geq c$; the two definitions of $\bar{g}_{r}$ agree on the overlap $\left[c, T_{1}\right]$. For $r \geq T_{2}$ we have $\bar{g}_{r}=e^{2 r} e^{2 c(k-1)} g_{B}$, i.e. $\bar{g}_{r}$ is the $e^{2 r}$ multiple of a fixed metric, as desired.

It remains to show that the sectional curvature $\bar{g}$ is almost in $\left[-k^{2},-1\right]$ for suitable $T_{1}, T_{2}$, and for this was essentially done in [BK05]. Indeed, if $r \leq T_{1}$, then $\bar{g}_{r}=g_{r}$, so [BK05] applies directly, and if $r \geq T_{1}$, then the proof in BK05] shows that up to a small error, the sectional curvature of $\bar{g}$ is expressed via the 
quantities $\left(\frac{h^{\prime}}{h}\right)^{2}=Q^{2}$ and $\frac{h^{\prime \prime}}{h}=Q^{\prime}+Q^{2}$, and the desired claim follows since $Q^{\prime}$ is small, and $Q \in[1, k]$.

\section{Circle bundles of type $(\mathrm{K})$}

In this section we describe some circle bundles whose total spaces lie in $\mathcal{B}$.

Proposition 6.1. The total space of an oriented circle bundle over closed nonpositively curved manifold has a nonpositively curved metric if and only if the bundle has torsion Euler class.

Proof. Consider an oriented (or equivalently principal) circle bundle with total space $E$ and base $M$.

For the "only if" direction recall that in $\mathrm{CAT}(0)$ groups centralizers virtually split [BH99, Theorem II.7.1(5)], so a fiber lifts homeomorphically to a finite cover $\hat{E}$ of $E$ where it represents the $\mathbb{Z}$-factor in the splitting $\pi_{1}(\hat{E}) \cong \mathbb{Z} \times H$ for some group $H$. It follows that the free circle action associated with the bundle lifts to a free circle action on $\hat{E}$, see [Bre72, Theorem I.9.1]. Thus $\hat{E}$ is the total space of an oriented circle bundle, and the covering $\hat{E} \rightarrow E$ descends to a map of orbit spaces $\hat{M} \rightarrow M$, so that the bundle structure on $\hat{E}$ is the pullback of the bundle structure on $E$. The bundle projection $\hat{E} \rightarrow \hat{M}$ maps $H$ isomorphically onto $\pi_{1}(\hat{M})$, and since $\hat{E}$ is aspherical, the bundle has a homotopy section, and hence its Euler class vanishes. Therefore, the rational Euler class of $E \rightarrow M$ vanishes because finite covers are injective on rational cohomology by a transfer argument.

For the "if" direction, note that vanishing of the rational Euler class forces the bundle to be flat Euclidean, see Miy01 or [OT05]. Thus its total space $E$ is a $\pi_{1}(M)$-quotient of $\widetilde{M} \times S^{1}$, where $\pi_{1}(M)$ acts by deck-transformations on the universal cover $\widetilde{M}$ and via a homomorphism $\pi_{1}(M) \rightarrow S^{1}$ on the second factor. The action is isometric, so the total space carries a metric of $K \leq 0$.

Remark 6.2. By the above proof, if the bundle has torsion Euler class, the metric of $K \leq 0$ on the total space has a local Euclidean de Rham factor, and hence the total space lies in $\mathcal{B}$ by Section 4 .

Deciding which circle bundles with non-torsion Euler class lie in $\mathcal{B}$ seems much harder; to date I can only handle type $(\mathrm{K})$ circle bundles, and the proof given below depends on one of the main results of [Bel12a].

We seek to construct a manifold $\mathbb{R} \times B$ as in Theorem 1.1(ii) such that $B$ is a circle bundle with non-torsion Euler class over a closed complex hyperbolic $(n-1)$-manifold $M$. Thus $M$ is the quotient of $\mathbf{C H}^{n-1}$ by a cocompact torsionfree lattice in $P U(n-1,1)$, where $\mathbf{C H}^{l}$ denotes the complex hyperbolic space of 
complex dimension $l$ normalized to have holomorphic sectional curvature -1 . The strategy is to realize $M$ as a totally geodesic submanifold in a complete complex hyperbolic $n$-manifold $V$ that is homotopy equivalent to $M$, and then modify the (incomplete) metric on $V \backslash M$ to make it as in Theorem 1.1(ii). Since there is no inclusion of $P U(n-1,1)$ into $P U(n, 1)$, in order to produce $V$ with desired properties we assume that $M$ has type (K), i.e. its holonomy representation $\pi_{1}(M) \rightarrow P U(n-1,1)$ lifts to $U(n-1,1)$, so that we can compose the lift with the inclusion into $U(n, 1)$ followed by the projectivization to $P U(n, 1)$.

To this end fix a torsion-free cocompact lattice in $U(n-1,1)$, where $n \geq 2$, and let $\hat{\Gamma}$ be the image of the lattice under the inclusion $U(n-1,1) \hookrightarrow U(n, 1)$. Let $\Gamma$ denote the projecton of $\hat{\Gamma}$ in $P U(n, 1)$, which acts on $\mathbf{C H}^{n}$ holomorphic isometries. The kernel of the projectivization $U(n, 1) \rightarrow P U(n, 1)$ consists of scalar matrices that form a diagonally embedded $U(1)$, so since $\hat{\Gamma}$ is discrete and torsion-free, the projection $\hat{\Gamma} \rightarrow \Gamma$ is an isomorphism.

Then $\Gamma$ acts freely on $\mathbf{C H}^{n}$, and it stabilizes a subspace $\mathbf{C} \mathbf{H}^{n-1} \subset \mathbf{C H}^{n-1}$ on which it acts with compact quotient $M:=\mathbf{C H}^{n-1} / \Gamma$; thus $M$ is a compact, totally geodesic, embedded submanifold of $\bar{M}:=\mathbf{C H}^{n} / \Gamma$. The metric on $\mathbf{C H}^{n}$ can be written in cylindrical coordinates about $\mathbf{C H}^{n-1}$ as $d r^{2}+g_{r}$ for $g_{r}=\sinh ^{2}(r) d \phi^{2}+\cosh ^{2}\left(\frac{r}{2}\right) \mathbf{k}^{n-1}$ where $r \geq 0$ and $\mathbf{k}^{n-1}$ is the standard metric on $\mathbf{C H}^{n-1}$, see [Bel12a, Section 3]. Let $F$ denote the unit normal bundle to $\mathbf{C H}^{n-1}$ in $\mathbf{C H}^{n}$, and set $\bar{F}:=F / \Gamma$. The proof of Bel12a, Theorem 1.1(iii)] yields a complete metric on $\mathbb{R} \times F$ of the form $d r^{2}+v^{2} d \phi^{2}+h^{2} \mathbf{k}^{n-1}$. The metric has sectional curvature in $[a, 0)$ for some negative constant $a$, and given $\varepsilon>0$ can be chosen to satisfy $v=\sinh (r)$ and $h=\cosh \left(\frac{r}{2}\right)$ for $r \geq \varepsilon$. The metric is invariant under the stabilizer of $\mathbf{C} \mathbf{H}^{n-1}$ in the isometry group of $\mathbf{C H}^{n}$, and hence it descends to a complete metric on $\mathbb{R} \times \bar{F}$, which we denote $g_{v, h}$. By construction in Bel12a] the portion $(-\infty, 0] \times \bar{F}$ has finite $g_{v, h}$-volume.

The subgroup $U(n-1,1)$ of $U(n, 1)$ commutes with the copy of $U(1)$ where $U(n-1,1) \times U(1)$ is embedded into $U(n, 1)$ via the map

$$
(A, B) \rightarrow\left[\begin{array}{rr}
A & 0 \\
0 & B
\end{array}\right],
$$

and hence their images in $P U(n, 1)$ also commute. It follows that there is an isometric $U(1)$-action on $\mathbf{C H}^{n}$ by rotation about $\mathbf{C} \mathbf{H}^{n-1}$, and the corresponding action on $\left(\mathbb{R} \times \bar{F}, g_{v, h}\right)$ is also isometric and free, where $U(1)$-orbits are the fibers of the normal circle bundles $\{r\} \times \bar{F} \rightarrow M$.

Let $\bar{F}_{m}$ denote the quotient of $\bar{F}$ by $\mathbb{Z}_{m} \leq U(1)$; this is a principal circle bundle over $M$ whose Euler class is the $m$ th multiple of the Euler class of $\bar{F}$. Let $\bar{g}_{v, h, m}$ denote the metric on $\mathbb{R} \times \bar{F}_{m}$ that is descended from $\left(\mathbb{R} \times \bar{F}, g_{v, h}\right)$; of course, $\bar{g}_{v, h, m}$ and $g_{v, h}$ are locally isometric. 
As is explained e.g. in Bel12a, Lemma 13.1] the first Chern class of normal bundle of $M$ in $\mathbf{C H}^{n} / \Gamma$ is represented $-\frac{\omega}{4 \pi}$ where $\omega$ is the Kähler form of the complex hyperbolic metric on $\mathbf{C H}^{n-1} / \Gamma$. Thus the Euler class of the circle bundle $\bar{F}_{m} \rightarrow M$ equals $-m \frac{\omega}{4 \pi}$; we refer to such circle bundles as the type (K).

Example 6.3. If $n=1$, then $M$ is a closed orientable surface of negative Euler characteristic, and according to [GKL01, Corollary 2.3.4] $-\frac{\omega}{4 \pi}$ integrated over $M$ equals $\pm \chi(M) / 2$; thus if $M$ has genus 2 , then every orientable circle bundle over $M$ has type $(\mathrm{K})$.

The metric $\left(\mathbb{R} \times \bar{F}_{m}, g_{v, h, m}\right)$ is simultaneously diagonalizable on the fiber, and it satisfies all properties needed in (ii) except that it is not equal to $d^{2}+e^{2 r} g_{B_{m}}$ for large $r$. In what follows we modify $g_{v, h, m}$ for large $r$ to make (ii) hold. The idea is similar to that of Section 5 but details are more involved. Locally

$$
\bar{g}_{v, h, m}=d r^{2}+v^{2} d \phi^{2}+h^{2} \mathbf{k}^{n-1}
$$

and for $r \geq \varepsilon$ we have $v=\sinh (r)$ and $h=\cosh \left(\frac{r}{2}\right)$, so the metric is complex hyperbolic, and its sectional curvature is within $\left[-1,-\frac{1}{4}\right]$. Small $C^{2}$ change of the warping functions affect the curvature only slightly, so as a first step we change $v, h$ so that $v=\frac{e^{r}}{2}$ and $h=\frac{1}{2} e^{\frac{r}{2}}$ for $r \geq T_{0}$ provided $T_{0}$ is large enough. Next we wish to change $h$ to $\frac{e^{r}}{2}$ for large $r$ while keeping curvature negative. To this end let $Q$ be a smooth non-decreasing function such that $Q=\frac{1}{2}$ on $\left[T_{0}, T_{1}\right]$ and $Q=1$ for $r \geq T_{2}$. Let $q(r):=\frac{T_{0}}{2}+\int_{T_{0}}^{r} Q(r) d r$. Setting $h:=\frac{e^{q}}{2}$ defines a metric, which we denote $g_{v, h, m}$, that agrees the previously defined metric for $r \leq T_{0}$, while if $r \geq T_{2}$, then $g_{v, h, m}-d r^{2}$ is the $e^{2 r}$ multiple of the fixed metric as desired for (ii).

It remains to show that the curvature remains negatively pinched for $r \geq T_{0}$. Set $s:=\frac{v}{h^{2}} ;$ as $h \geq \frac{1}{2} e^{\frac{r}{2}}$ we get $0<s \leq 2$ for $r \geq T_{0}$. The sectional curvature of any metric of the form $g_{v, h, m}$ was computed in [Bel12a, Section 9] in terms of $h$ and $v$, and below we review the results of this computation. Let $\{C, D\}$ denote an orthonormal basis in a two-plane with

$$
C=c_{0} \partial_{r}+c_{1} Y_{1}+c_{2} Y_{2}+c_{3} Y_{3}, \quad D=d_{1} Y_{1}+d_{2} Y_{2}
$$

where $c_{i}, d_{j} \in \mathbb{R}$ and $\left\{\partial_{r}, Y_{1}, Y_{2}, Y_{3}\right\}$ are orthonormal, and $Y_{1}$ is tangent to the circle fiber. The sectional curvature of the plane is then given by

$$
\begin{aligned}
& \left(d_{1} c_{2}-d_{2} c_{1}\right)^{2} K\left(Y_{2}, Y_{1}\right)+d_{1}^{2} c_{3}^{2} K\left(Y_{3}, Y_{1}\right)+d_{1}^{2} c_{0}^{2} K\left(\partial_{r}, Y_{1}\right)+ \\
& d_{2}^{2} c_{0}^{2} K\left(\partial_{r}, Y_{2}\right)+d_{2}^{2} c_{3}^{2} K\left(Y_{3}, Y_{2}\right)+3 d_{1} d_{2} c_{0} c_{3}\left\langle R\left(\partial_{r}, Y_{1}\right) Y_{2}, Y_{3}\right\rangle
\end{aligned}
$$


where

$$
\begin{gathered}
K\left(Y_{2}, Y_{1}\right)=K\left(Y_{3}, Y_{1}\right)=\frac{s^{2}}{16}-\frac{v^{\prime}}{v} \frac{h^{\prime}}{h} \leq \frac{1}{4}-Q \leq-\frac{1}{4}, \\
K\left(Y_{3}, Y_{2}\right)=-\frac{1}{4 h^{2}}-\frac{3}{h^{2}} c_{23}^{2}-3 c_{23}^{2} \frac{s^{2}}{4}-\left(\frac{h^{\prime}}{h}\right)^{2}<-3 c_{23}^{2} \frac{s^{2}}{4}, \\
K\left(\partial_{r}, Y_{1}\right)=-\frac{v^{\prime \prime}}{v}=-1, \quad K\left(\partial_{r}, Y_{2}\right)=-\frac{h^{\prime \prime}}{h}=-Q^{\prime}-Q^{2}, \\
\left\langle R\left(\partial_{r}, Y_{1}\right) Y_{2}, Y_{3}\right\rangle=-c_{23} \frac{v}{h^{2}}\left(\frac{v^{\prime}}{v}-\frac{h^{\prime}}{h}\right)=-c_{23} s(1-Q) .
\end{gathered}
$$

where $c_{23}$ is a constant with $\left|c_{23}\right| \leq \frac{1}{2}$ defined by $\left[Y_{2}, Y_{3}\right]=c_{23} \frac{v}{h^{2}} Y_{1}$. Now

$$
\begin{gathered}
d_{1}^{2} c_{0}^{2} K\left(\partial_{r}, Y_{1}\right)+d_{2}^{2} c_{3}^{2} K\left(Y_{3}, Y_{2}\right)<-\left|d_{1} c_{0}\right|^{2}-\left|d_{2} c_{3}\right|^{2} \frac{3 c_{23}^{2} s^{2}}{4}= \\
-\left(\left|d_{1} c_{0}\right|-\left|d_{2} c_{3}\right| \frac{\sqrt{3}\left|c_{23}\right| s}{2}\right)^{2}-\left|d_{1} c_{0} d_{2} c_{3} c_{23}\right| \sqrt{3} s,
\end{gathered}
$$

while $0 \leq 1-Q \leq \frac{1}{2}$ implies

$$
3 d_{1} d_{2} c_{0} c_{3}\left\langle R\left(\partial_{r}, Y_{1}\right) Y_{2}, Y_{3}\right\rangle \leq\left|d_{1} d_{2} c_{0} c_{3} c_{23}\right| \frac{3}{2} s
$$

so these three terms of (6.4) add up to a nonpositive number. Since the other three terms are also nonpositive, $K(C, D) \leq 0$, and if $K(C, D)$ is zero somewhere, then the coefficients for the latter three terms vanish, and in particular, $d_{1} c_{3}=0$, so the mixed term $3 d_{1} d_{2} c_{0} c_{3}\left\langle R\left(\partial_{r}, Y_{1}\right) Y_{2}, Y_{3}\right\rangle$ is not present in the sum, and elementary linear algebra (as in Bel12a, Remark 9.6]) leads to a contradiction with $K(C, D)=0$. Thus $K(C, D)<0$ for $r \geq T_{0}$, which completes the proof that every type $(\mathrm{K})$ circle bundle lies in $\mathcal{B}$.

Remark 6.5. A similar construction in the real hyperbolic case yields circle bundles with torsion Euler class, see Bel12b.

\section{Proof of Theorem 1.2}

That $\mathcal{B}$ contains each infranilmanifold, every circle bundle of type $(\mathrm{K})$, and each closed manifold of $K \leq 0$ with a local Euclidean de Rham factor is proved in Sections 46 and in each case the metric on the the fiber $B$ is simultaneously diagonalizable. Hence Section 3 implies that $\mathcal{B}$ is closed under products, and obviously it is closed under disjoint unions. Finally, if $B$ is a manifold in $\mathcal{B}$, then inductively it comes with a family $g_{r}$ of simultaneously diagonalizable metrics such that $d r^{2}+g_{r}$ is as in (ii). If $\left(M, g_{M}\right)$ is a closed manifold of $K \leq 0$, and $h$ is as in Theorem 4.1, then $g_{r}+h^{2} g_{M}$ is simultaneously diagonalizable, and $d r^{2}+g_{r}+h^{2} g_{M}$ is as in (ii). 


\section{REFERENCES}

[AS92] U. Abresch and V. Schroeder, Graph manifolds, ends of negatively curved spaces and the hyperbolic 120 -cell space, J. Differential Geom. 35 (1992), no. 2, 299-336.

[Bel12a] I. Belegradek, Complex hyperbolic hyperplane complements, Math. Ann. 353 (2012), no. $2,545-579$.

[Bel12b] , Rigidity and relative hyperbolicity of real hyperbolic hyperplane complements, Pure Appl. Math. Q. 8 (2012), no. 1, 15-51.

[Bes08] A. L. Besse, Einstein manifolds, Classics in Mathematics, Reprint of the 1987 edition, Springer-Verlag, Berlin, 2008.

[BFL] A. Bartels, F. T. Farrell, and W. Lueck, The Farrell-Jones conjecture for cocompact lattices in virtually connected Lie groups, arXiv:1101.0469 1.

[BGS85] W. Ballmann, M. Gromov, and V. Schroeder, Manifolds of nonpositive curvature, Progress in Mathematics, vol. 61, Birkhäuser Boston Inc., Boston, MA, 1985.

[BH99] M. Bridson and A. Haefliger, Metric spaces of non-positive curvature, Grundlehren der Mathematischen Wissenschaften [Fundamental Principles of Mathematical Sciences], vol. 319, Springer-Verlag, Berlin, 1999.

[BK05] I. Belegradek and V. Kapovitch, Pinching estimates for negatively curved manifolds with nilpotent fundamental groups, Geom. Funct. Anal. 15 (2005), no. 5, 929-938.

[BL] A. Bartels and W. Lueck, The Borel Conjecture for hyperbolic and CAT(0)-groups, arXiv:0901.0442 2 .

[BP] I. Belegradek and T. T. Nguyen Phan, Non-aspherical ends and nonpositive curvature, arXiv: 1212.3303 v 1.

[Bre72] G. E. Bredon, Introduction to compact transformation groups, Pure and Applied Mathematics, vol. 46, Academic Press, New York, 1972.

[Buy93] S. V. Buyalo, An example of a four-dimensional manifold of negative curvature, Algebra i Analiz 5 (1993), no. 1, 193-199.

[BW04] I. Belegradek and G. Wei, Metrics of positive Ricci curvature on bundles, Int. Math. Res. Not. (2004), no. 57, 3079-3096.

[CD95] R. M. Charney and M. W. Davis, Strict hyperbolization, Topology 34 (1995), no. 2, 329-350.

[Fuj88] K. Fujiwara, A construction of negatively curved manifolds, Proc. Japan Acad. Ser. A Math. Sci. 64 (1988), no. 9, 352-355.

[GKL01] W. M. Goldman, M. Kapovich, and B. Leeb, Complex hyperbolic manifolds homotopy equivalent to a Riemann surface, Comm. Anal. Geom. 9 (2001), no. 1, 61-95.

[Gro78] M. Gromov, Manifolds of negative curvature, J. Differential Geom. 13 (1978), no. 2, $223-230$.

[Gro82] , Volume and bounded cohomology, Inst. Hautes Études Sci. Publ. Math. (1982), no. 56, 5-99 (1983).

[Miy01] S. Miyoshi, A remark on torsion Euler classes of circle bundles, Tokyo J. Math. 24 (2001), no. 1, 189-194.

[Ont] P. Ontaneda, Pinched smooth hyperbolization, arXiv:1110.6374 1.

[OT05] J. Oprea and D. Tanré, Flat circle bundles, pullbacks, and the circle made discrete, Int. J. Math. Math. Sci. (2005), no. 21, 3487-3495.

[Pha] T. T. Nguyen Phan, Nil happens. what about Sol?, arXiv:1207.1734v1.

Igor Belegradek, School of Mathematics, Georgia Institute of Technology, AtLANTA, GA 30332-0160

E-mail address: ib@math.gatech.edu 